\title{
STABLE FILTERING FOR ARTIFICIAL PHEROMONE POTENTIAL FIELD CONSTRUCTED BY MOBILE ROBOTS AND PASSIVE RFID TAGS
}

\author{
Piljae $\mathrm{Kim}^{1}$ and Daisuke Kurabayashi ${ }^{2}$ \\ Department of Mechanical and Control Engineering, Tokyo Institute of Technology, 2- \\ 12-1 Ookayama, Meguro-ku, Tokyo 152-8552, Japan \\ 1 pjkimeirs.ctrl.titech.ac.jp \\ ${ }^{2}$ dkuraeirs.ctrl.titech.ac.jp
}

\begin{abstract}
In this study, the concept of the chemical substance pheromone is utilized for the robotic tasks. This paper first illustrates the model of pheromone-based potential field. The field is constructed through the interaction between mobile robots and data carriers, such as RFID tags. The emphasis in the modeling of the system is on the possibility of the practical implementable ideas. The stability analysis of the pheromone potential field is carried out also aiming at the implementation on a real robotic environment. The comprehensive analysis on stability provides the criteria for how the parameters are to be set for the proper potential field, and has led to a new filter design scheme called pheromone filter, which satisfies both the stability and accuracy of the field. The unique structures of both the revised mobile robot and the designed filter show that the proposed method facilitates a more straightforward and practical implementation.
\end{abstract}

\section{KEYWORDS}

Pheromone potential field, Mobile robots, RFID tags, Stability analysis, Pheromone filter

\section{INTRODUCTION}

It has been known for some time that social insects such as ants and bees communicate with each other through a process which is generally called stigmergy, and perform given tasks effectively by using the chemical substance pheromone [1]. Although the term stigmergy was originally introduced to explain the behavior of termite societies, it later came to describe indirect communication mediated by modifications of the environment that can also be observed in other social insects [2]. Inspired from these biological characteristics, researchers have been recently motivated to undertake studies on pheromone-based robotics [3, 4]. In addition to this, there are other studies that make use of the RFID (Radio-Frequency Identification) technology [5] for realizing digital or artificial pheromones [6,7], wherein the agents communicate with other agents by updating a pheromone trail through the RFID tags distributed in an environment.

In particular, interests in RFID technology for navigation of the mobile robot have been currently growing [8, 9]. For instance, Vorst et al. focused on SLAM (Simultaneous Localization And Mapping) techniques that map static tags' locations [10], and Kodaka et al. tried to build a navigational entropy map using RFID tags distributed on the floor [11]. However, few researches have suggested guidelines on stability when applying RFID tags for these real robotic tasks. 
This study utilizes the idea of the artificial pheromone. The study emphasizes that the stability analysis is fundamental not only to secure the simple implementation, but also to improve the scheme in both stability and accuracy.

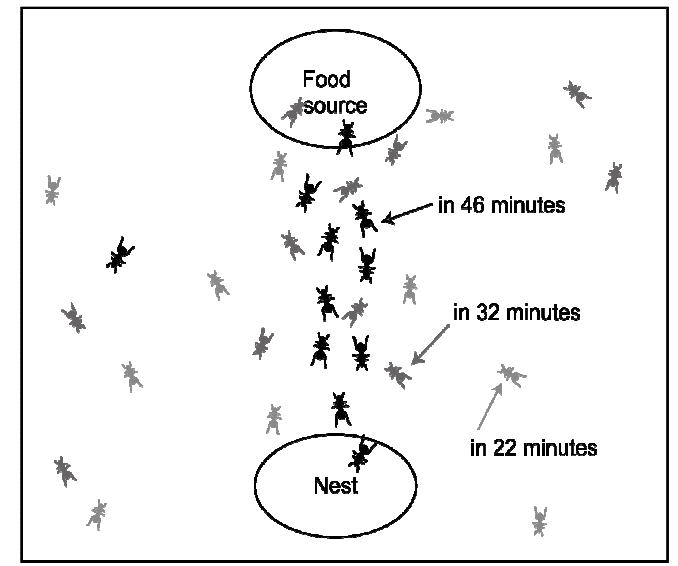

(a) The shortcut between food source and nest in ant colony

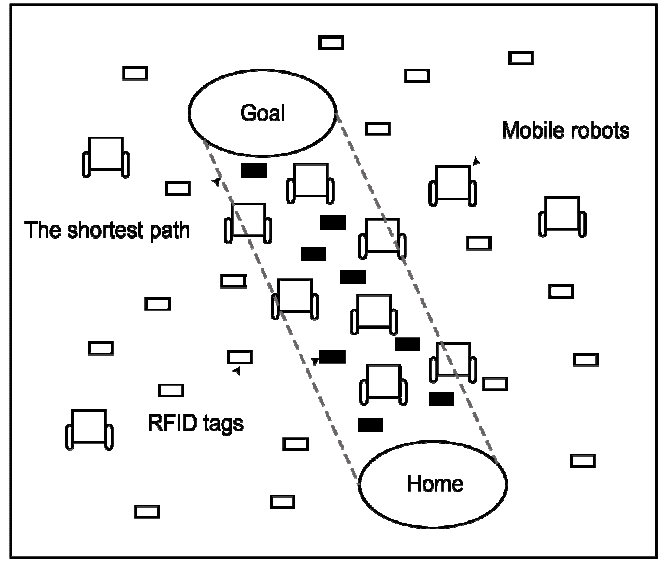

(b) The shortest path between goal and home in robotic platform

Figure 1. The shortcut on which pheromone is accumulated in the Lasius niger colony (adapted from Camazine et al. [12]) and its realization in robotic platform: the darker objects indicate the ants observed from more elapsed time in (a), and the black-filled RFID tags represent those storing the information of artificial pheromone in (b)

This paper first illustrates the scheme of pheromone-based interaction. Passive RFID tags are spread out onto the floor so that they can be used as storage of the field information. The artificial pheromone is effectively distributed over the whole field via the communication between robots and RFID tags. The next section details the deployment of the pheromone, thereby highlighting how this process can be considered to be similar to the spatial smoothing of the image plane, which is typically realized by the masked kernel. After the observation of the models, the stability criteria are formulated and evaluated in section 3, where the necessity of the less restricted algorithm is raised. As a consequence, the implicit method is introduced in section 4. All of these investigations will be concluded as the designing scheme of a new filter in section 5 , where a proposed method provides more freedom for designing a kernel. Some closing remarks will be made in the last section.

\section{MODELING BASED ON PHEROMONE DEPLOYMENT}

In this section, we outline the model and present the real platform that is being developed. Let us first introduce the ant colony as the most popular biological model that makes entire use of the pheromone.

Figure 1(a) shows the shortcut-producing process observed from the black garden ant (Lasius niger) colony, where the distance between the food source and the nest is set 1 meter and ants were randomly distributed at the beginning of the experiment. When a colony is offered a food source, a scouting ant discovers the source and returns to the nest, laying a pheromone trail which dissipates over time and distance. At the nest, other foraging ants detect the trail and follow it to the food source. Those that arrive at the source load food and return to the nest, thereby reinforcing the trail. By contrast, unsuccessful pheromone trail-followers explore the field before either returning to the nest or finding the food source. Since the trail is reinforced, more ants are recruited in accordance with the time, and as a result, the shortcut between the 
food source and the nest is formed by the strengthened pheromone trail. Likewise, if there are sufficient number of robots and data storing devices such as RIFD tags, the robots may also form and follow the shortest path between home and goal as shown in Figure 1(b), which is the

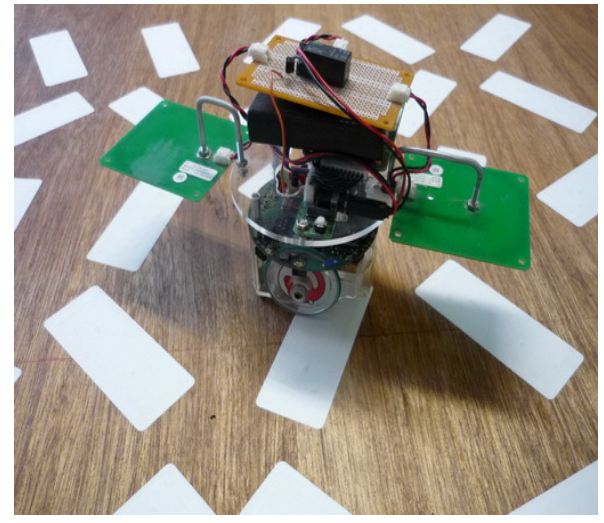

(a) Revised e-puck robot with RFID reader and writer and RFID tags distributed on the floor

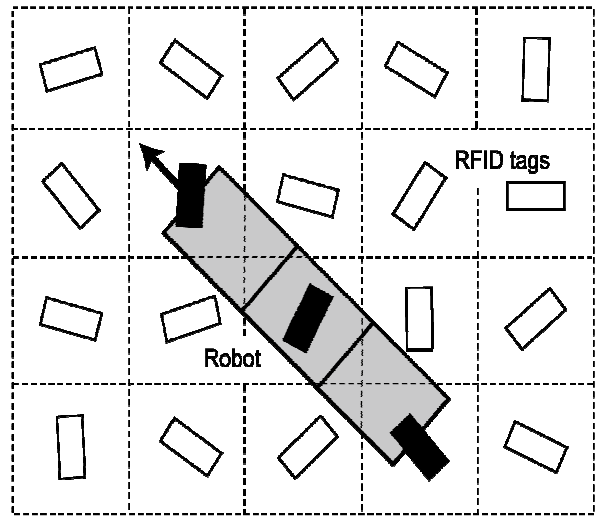

(b) Concept of the interaction between a mobile robot and RFID tags

Figure 2. The revised robot and the illustration of the interaction in the experimental field: in (b), the arrow denotes the heading of the robot and the black-filled RFID tags indicate those to be updated

fundamental concept of pheromone-based robotics. How, then, can the numbers of the robots and RFID tags be determined so that the system works properly? Also, what kinds of functions are required for each agent? This study has been launched in order to provide an answer for these questions.

In the framework of this study, the system illustrated in Figure 1(b) can be realized via the interaction between mobile agents with RFID transceivers and RFID tags, on which the digital equivalent of pheromones is laid by the agents.

For mobile agents, we use revised e-puck robots that have RFID readers and writers on their forward and backward side, as shown in Figure 2(a). The passive RFID tags, which are the white rectangular tags in Figure 2(a), are adopted for this study in the same way as our previous work [7]. The RFID tags are wireless and battery-free, and each tag is marked with a unique identifier and is equipped with a small memory that allows it to store data. The data consists of the tags' own IDs and scalar pheromone values. The passive nature of the RFID tags implies that pheromone can be diffused only via the interaction between robots and RFID tags, which is illustrated in Figure 2(b).

If we assume that the RFID tags are uniformly distributed over the whole field, the concept of the pheromone deployment is equivalent to the smoothing or blurring of a digital image. In other words, since the spatial filtering process consists simply of moving the filter mask from point to point in an image, the diffusion of the pheromone over the field of the RFID tags using a mobile robot can be considered as a filtering operation of the image. In this mechanic, the robot corresponds to the filter mask and the RFID tags act as pixel points. This paper evaluates and develops all algorithms on the assumption that the field consists of a uniform lattice. Note that, from Figure 2, the structure of the revised robot is fairly similar in shape to the onedimensional filter mask. By virtue of this unique feature, it is expected to be adequately 
International Journal of Computer Science \& Information Technology (IJCSIT) Vol 3, No 5, Oct 2011

straightforward to implement an algorithm after evaluation. We will thoroughly use this structural concept when developing a new scheme, pheromone filter, in the latter section. The filter is to be implemented on the mobile robot as an action algorithm so that the artificial pheromone is propagated through the RFID tags in a stable way.

\section{Pheromone POTENTIAL Field}

\subsection{Stability condition of the model}

The mathematical pheromone model in one-dimensional space $x$ is

$$
\frac{\partial u(t, x)}{\partial t}=D \frac{\partial^{2} u(t, x)}{\partial x^{2}}-K u(t, x)
$$

where the coefficients $D$ and $K$ represent diffusion and evaporation rate respectively [13]. We can easily extend the same idea to the two-dimensional space without losing generality, for example, the pheromone model in two-dimensional space $(x, y)$ is

$$
\frac{\partial u(t, x, y)}{\partial t}=D\left(\frac{\partial^{2} u(t, x, y)}{\partial x^{2}}+\frac{\partial^{2} u(t, x, y)}{\partial y^{2}}\right)-K u(t, x, y) .
$$

This paper, therefore, focuses on the one-dimensional case for reasons of simplicity. Although there are several solutions for this sort of PDE (Partial Differential Equation) problem, this study adopts the finite difference calculus method, because it is considered to be the most suitable for implementation on a real robotic environment. Equation (1) can be discretized as following difference equation through using the FTCS (Forward Time Central Space) scheme

$$
\begin{aligned}
& \frac{1}{\Delta t}\left\{u\left(t_{n}+\Delta t, x_{i}\right)-u\left(t_{n}, x_{i}\right)\right\} \\
& =\frac{D}{(\Delta x)^{2}}\left\{u\left(t_{n}, x_{i}-\Delta x\right)-2 u\left(t_{n}, x_{i}\right)+u\left(t_{n}, x_{i}+\Delta x\right)\right\}-K u\left(t_{n}, x_{i}\right),
\end{aligned}
$$

where we calculate the time derivative in a forward manner and calculate the space derivative in a centered manner.

The numerical stability is estimated with relation to the growth or decrease of the rounding error in the calculation scheme of the finite difference method. In this study, we consider the perturbation stability analysis [14], which is, in our opinion, the simplest and most straightforward.

Let us slightly simplify the equations by using the notation that temporal indices are represented by a superscript and spatial indices are represented by a subscript, such that the value of the function $U$ at the time $t_{n}$ and at the point $x_{i}$ is expressed as $U_{i}^{n}$, i.e., $u\left(t_{n}, x_{i}\right)=U_{i}^{n}$.

The pheromone equation using this notation becomes

$$
\frac{1}{\Delta t}\left\{U_{i}^{n+1}-U_{i}^{n}\right\}=\frac{D}{(\Delta x)^{2}}\left\{U_{i-1}^{n}-2 U_{i}^{n}+U_{i+1}^{n}\right\}-K U_{i}^{n} .
$$

If we add the perturbation $\varepsilon_{i}^{n}$ to around $U_{i}^{n}$, the difference equation is written

$$
\frac{1}{\Delta t}\left\{U_{i}^{n+1}-\left(U_{i}^{n}+\varepsilon_{i}^{n}\right)\right\}=\frac{D}{(\Delta x)^{2}}\left\{U_{i-1}^{n}-2\left(U_{i}^{n}+\varepsilon_{i}^{n}\right)+U_{i+1}^{n}\right\}-K\left(U_{i}^{n}+\varepsilon_{i}^{n}\right) .
$$


International Journal of Computer Science \& Information Technology (IJCSIT) Vol 3, No 5, Oct 2011

Having rearranged the equation, we get

$$
U_{i}^{n+1}=\underbrace{(1-K \Delta t) U_{i}^{n}+\frac{D \Delta t}{(\Delta x)^{2}}\left\{U_{i-1}^{n}-2 U_{i}^{n}+U_{i+1}^{n}\right\}}_{\hat{U}_{i+1}^{n}}+\underbrace{\varepsilon_{i}^{n}\left\{1-\frac{2 D \Delta t}{(\Delta x)^{2}}-K \Delta t\right\}}_{\varepsilon_{i}^{n+1}} .
$$

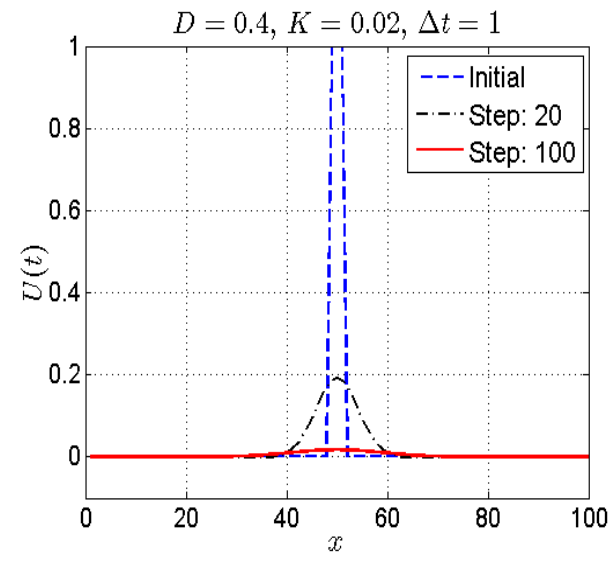

(a) Stable case

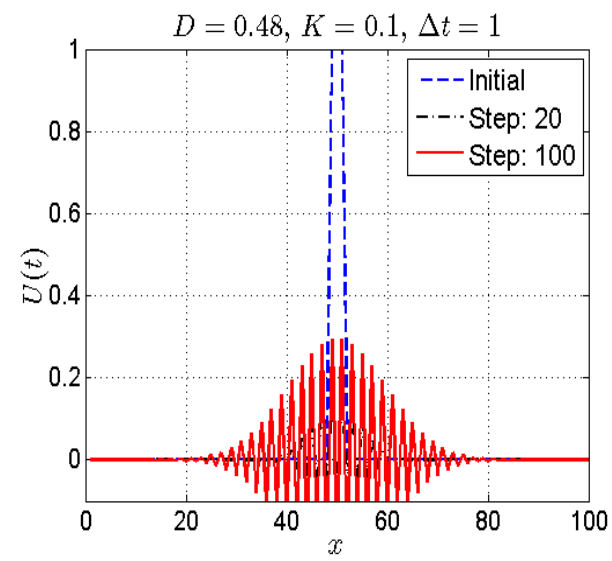

(c) Unstable for large $K$

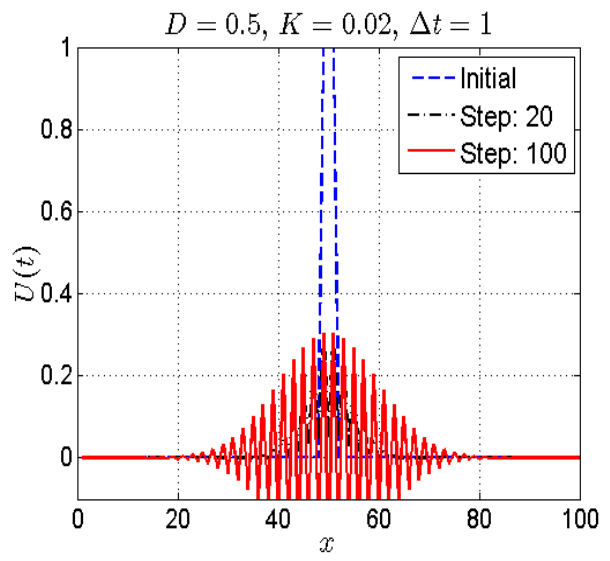

(b) Unstable for large $D$

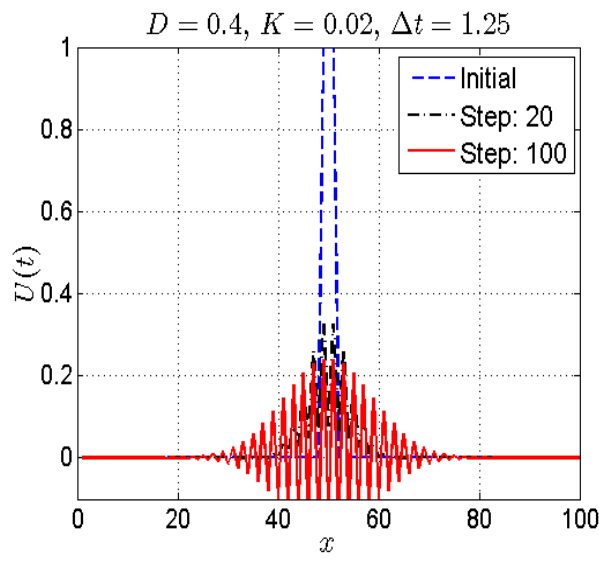

(d) Unstable for large $\Delta t$

Figure 3. The outputs of the pheromone equation for varying parameters

For the stable behavior of the system, the perturbation should be decreased, which means the condition

$$
\left|\frac{\varepsilon_{i}^{n+1}}{\varepsilon_{i}^{n}}\right|<1
$$

must hold. In view of the case of no overshoot, the above condition should be changed as 


$$
0 \leq\left|\frac{\varepsilon_{i}^{n+1}}{\varepsilon_{i}^{n}}\right|<1 .
$$

From Equation (6) and (8), the stability condition without overshoot leads to

$$
\left(\frac{D}{(\Delta x)^{2}}+\frac{K}{2}\right) \Delta t \leq \frac{1}{2}
$$

For a two-dimensional case, if a square cell is assumed, the following condition is required for each $x$ and $y$ direction.

$$
\left(\frac{D}{(\Delta x)^{2}}+\frac{K}{4}\right) \Delta t \leq \frac{1}{4}, \quad\left(\frac{D}{(\Delta y)^{2}}+\frac{K}{4}\right) \Delta t \leq \frac{1}{4}
$$

\subsection{Numerical simulation}

From the derived stability condition, it is noted that all varying parameters, i.e., $K, D, \Delta t$ and $\Delta x$, are coupled together for the stable solution, thus we performed a numerical simulation in a leave-one-out cross validation way. That is, we observe the outputs as varying one coefficient while keeping all other parameters the same. The only parameter $\Delta x$ is fixed as $\Delta x=1$ for displaying the simulation results in a more comprehensible way. In addition, throughout the simulations presented in this paper, we assign constant boundary conditions at the left-hand and right-hand edges, i.e., the Dirichlet boundary conditions are imposed.

Figure 3 shows the outputs of the pheromone equation. Unstable behaviors are observed for the larger $D, K$ and $\Delta t$ than the formulated criterion (9). The results of the simulation indicate that the derived stability condition works properly, and, what is more, illustrate that all parameters contribute strictly and independently to the stability of the potential field. It is obvious that $\Delta x$ and $\Delta t$ are critical coefficients, especially in pheromone-based implementation, because updating the frequency of the potential field depends mainly on the number of RFID tags and the speed of the robots.

Unfortunately, however, it is not possible to precisely configure these parameters in advance, partly because of the inherent uncertainty of the real experimental environment, and partly because of the highly-coupled properties of each parameter in the stability condition.

From this point on, we use $D=0.4, K=0.02, \Delta t=1$ and $\Delta x=1$ as typical stable parameters. The parameters that consist of the unstable cases for large $K$ and $\Delta t$, i.e., Figure 3(c) and Figure 3(d), will be used when comparing the stability of the other schemes in the next section.

\section{STABLE SOLUTION FOR THE PHEROMONE MODEL}

When applying a pheromone model to the real robotic system, as we have examined, all parameters consisting of stability condition need to be carefully designed, otherwise they can be a bottleneck. To relax these restrictions, we have noticed the fact that the implicit time stepping can improve or even eliminate stability limitations, which suggests that combining a backward scheme in time with a central difference approximation in space, i.e., the so-called BTCS (Backward Time Central Space) scheme, may make a pheromone potential field more stable.

\subsection{BTCS scheme}

The BTCS scheme can be written 
International Journal of Computer Science \& Information Technology (IJCSIT) Vol 3, No 5, Oct 2011

$$
\frac{1}{\Delta t}\left\{U_{i}^{n+1}-U_{i}^{n}\right\}=\frac{D}{(\Delta x)^{2}}\left\{U_{i-1}^{n+1}-2 U_{i}^{n+1}+U_{i+1}^{n+1}\right\}-K U_{i}^{n+1},
$$

which is logical and obvious from the definition of the scheme. If we define diffusion number and evaporation number as $d=\frac{D \Delta t}{(\Delta x)^{2}}$ and $k=-\Delta t K$, respectively, the equation is written

$$
\left\{U_{i}^{n+1}-U_{i}^{n}\right\}=d\left\{U_{i-1}^{n+1}-2 U_{i}^{n+1}+U_{i+1}^{n+1}\right\}-k U_{i}^{n+1}
$$

There is a general knowledge that the Fourier coefficient of the solution of Equation (12) can be written

$$
U_{i}^{n}=V^{n} e^{j \omega_{x}(i \Delta x)}
$$

where $j=\sqrt{-1}$, and $V^{n}$ is the amplitude of the $n$th harmonic. If we set phase angle $\theta=\omega_{x} \Delta x$, $U_{i}^{n}$ and $U_{i \pm 1}^{n+1}$ can be described as the following Fourier series.

$$
U_{i}^{n}=V^{n} e^{j i \theta}, \quad U_{i \pm 1}^{n+1}=V^{n+1} e^{j(i \pm) \theta}
$$

Let us substitute Equation (14) into Equation (12) and divide by $e^{j i \theta}$, then

$$
V^{n+1}-V^{n}=d V^{n+1}\left(e^{j \theta}+e^{-j \theta}-2\right)-k V^{n+1}
$$

is derived. Using the Euler's formula, the equation is rearranged as

$$
V^{n+1}(1+2 d(1-\cos \theta)+k)=V^{n} .
$$

If we define an amplification factor $G$ as

$$
V^{n+1}=\frac{1}{(1+2 d(1-\cos \theta)+k)} V^{n}=G V^{n}
$$

the condition

$$
|G|=\left|\frac{1}{(1+2 d(1-\cos \theta)+k)}\right| \leq 1
$$

must apply for the converging solution. This inequality is called the von Neumann stability condition. From the fact that $d>0,(1-\cos \theta) \geq 0$ and $k>0$, the condition (18) is always the case. This means that the BTCS scheme is unconditionally stable for any parameters.

If we define diffusion matrix $\mathbf{D}_{\mathbf{m}}$ as 


$$
\mathbf{D}_{\mathbf{m}}=\left(\begin{array}{ccccc}
-2 & 1 & 0 & \cdots & 0 \\
1 & -2 & 1 & \cdots & 0 \\
0 & 1 & -2 & \cdots & 0 \\
\vdots & \vdots & \vdots & \ddots & \vdots \\
0 & 0 & 0 & \cdots & -2
\end{array}\right)
$$

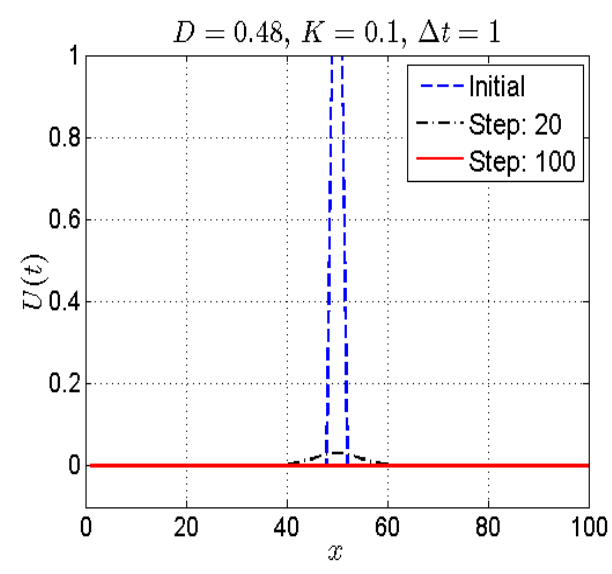

(a) Stable for large $K$

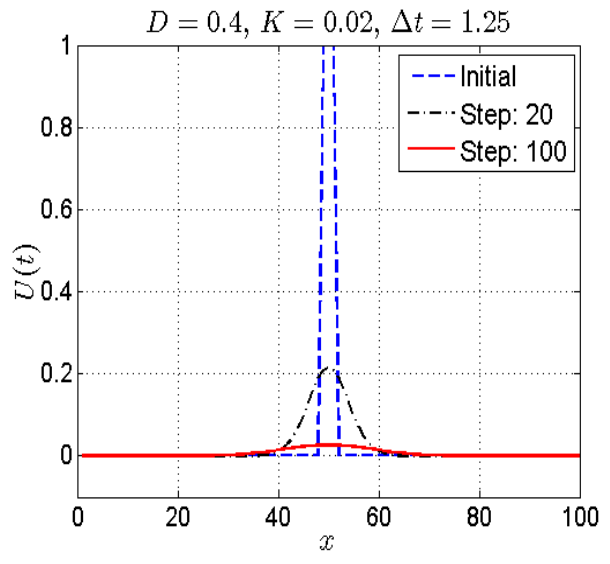

(b) Stable for large $\Delta t$

Figure 4. The outputs of the pheromone equation for varying parameters using the BTCS scheme

and represent Equation (12) in matrix form, the scheme is written as the following simplified form.

$$
\begin{aligned}
& \mathbf{U}^{n+1}=\mathbf{U}^{n}+d \mathbf{D}_{\mathbf{m}} \mathbf{U}^{n+1}-k \mathbf{U}^{n+1} \\
& \left(\mathbf{I}-d \mathbf{D}_{\mathbf{m}}+k \mathbf{I}\right) \mathbf{U}^{n+1}=\mathbf{U}^{n}
\end{aligned}
$$

Note that the rank of $\left(\mathbf{I}-d \mathbf{D}_{\mathbf{m}}+k \mathbf{I}\right)$ is never reduced, hence the solution

$$
\mathbf{U}^{n+1}=\left(\mathbf{I}-d \mathbf{D}_{\mathbf{m}}+k \mathbf{I}\right)^{-1} \mathbf{U}^{n}
$$

always exists. The stable behavior can be observed from Figure 4 in comparison with the output under the same condition as that used for Figure 3 . We present only the case for the large $K$ and $\Delta t$ parameters, at which the output appeared unstable when using the FTCS scheme.

\subsection{Crank-Nicolson scheme}

As far as stability is concerned, we are definitely able to get a solution for the pheromone equation. We also anticipate that the computational cost is not that expensive, because $\left(\mathbf{I}-d \mathbf{D}_{\mathbf{m}}+k \mathbf{I}\right)$ forms a tri-diagonal matrix [15]

However, when it comes to the implementation of Equation (20), we encounter with a crucial difficulty, due to the fact that the inverse of a tri-diagonal matrix is no longer tri-diagonal. That is, most of the elements are required in order to retrieve the temporal and spatial information 
from an inverse matrix. Also, regarding the accuracy of the BTCS scheme, since it is a temporal approximation, the method contains more numerical errors than the FTCS scheme.

On the basis of these considerations, the Crank-Nicolson method was deemed to be the most appropriate solution for this study. It is second-order and implicit in time, and is numerically stable [16]. The method was proposed mainly to improve the accuracy of the BTCS scheme, but we are, rather, interested in the structure produced by the method. It has the averaged form of the FTCS and BTCS schemes at $n$ and $n+1$, respectively.

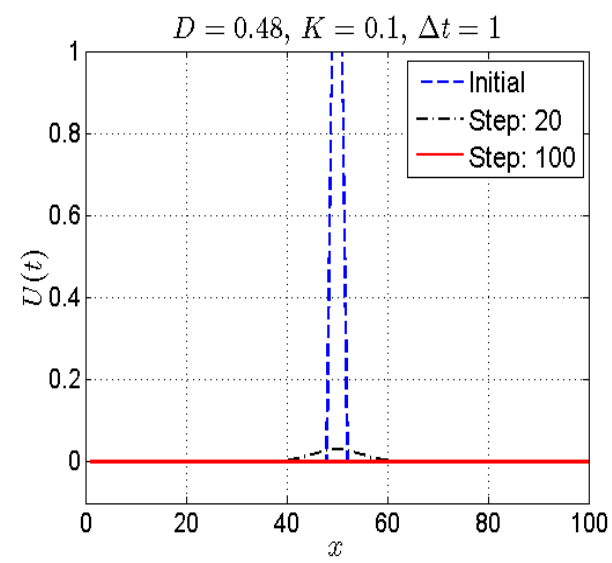

(a) Stable for large $K$

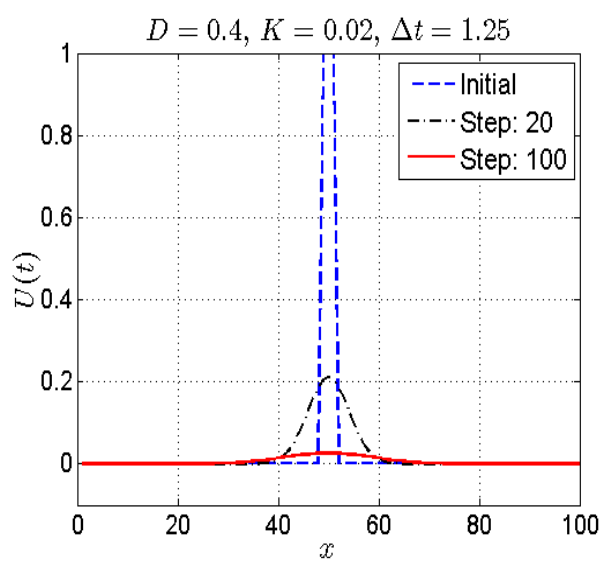

(b) Stable for large $\Delta t$

Figure 5. The outputs of the pheromone equation for varying parameters using the CrankNicolson scheme

$$
\frac{1}{\Delta t}\left(U_{i}^{n+1}-U_{i}^{n}\right)=\frac{D}{2}\left(\frac{U_{i-1}^{n}-2 U_{i}^{n}+U_{i+1}^{n}}{(\Delta x)^{2}}+\frac{U_{i-1}^{n+1}-2 U_{i}^{n+1}+U_{i+1}^{n+1}}{(\Delta x)^{2}}\right)-\frac{K}{2}\left(U_{i}^{n}+U_{i}^{n+1}\right)
$$

The von Neumann stability condition is obtained as

$$
V^{n+1}=\frac{(1-2 d(1-\cos \theta)-k)}{(1+2 d(1-\cos \theta)+k)} V^{n}=G V^{n}
$$

using the same technique that was used for the BTCS scheme. From the above condition, it is obvious that the value $|G|$ will not go higher than 1, and thus the Crank-Nicolson scheme is unconditionally stable for all varying parameters. The stability is validated in Figure 5.

Let us finally represent the method as a matrix form in order to clarify the mathematical structure.

$$
\begin{aligned}
\mathbf{U}^{n+1} & =\left(\mathbf{I}-d \mathbf{D}_{\mathbf{m}}+k \mathbf{I}\right)^{-1}\left(\mathbf{I}+d \mathbf{D}_{\mathbf{m}}-k \mathbf{I}\right)^{-1} \mathbf{U}^{n} \\
& =\mathbf{W} \mathbf{U}^{n}
\end{aligned}
$$

It is worthwhile to mention here that the system (23) is stable either when

$$
\|\mathbf{W}\|<1
$$


where $\|\mathbf{W}\|$ represents Euclidean norm, or

$$
\left.\begin{array}{cccccccccc}
\multicolumn{10}{c}{\mu_{\mathrm{w}}=\max _{1 \leq j \leq n}\left|\lambda_{j}\right| \leq 1,} \\
0.44 & 0.21 & 0.03 & 0.00 & 0.00 & 0.00 & 0.00 & 0.00 & 0.00 & 0.00 \\
0.21 & 0.47 & 0.22 & 0.03 & 0.00 & 0.00 & 0.00 & 0.00 & 0.00 & 0.00 \\
0.03 & 0.22 & 0.47 & 0.22 & 0.03 & 0.00 & 0.00 & 0.00 & 0.00 & 0.00 \\
0.00 & 0.03 & 0.22 & 0.47 & 0.22 & 003 & 0.00 & 0.00 & 0.00 & 0.00 \\
0.00 & 0.00 & 0.03 & 0.22 & 0.47 & 0.22 & 0.03 & 0.00 & 0.00 & 0.00 \\
0.00 & 0.00 & 0.00 & 0.03 & 0.22 & 0.47 & 0.22 & 0.03 & 0.00 & 0.00 \\
0.00 & 0.00 & 0.00 & 0.00 & 0.03 & 0.22 & 0.47 & 0.22 & 0.03 & 0.00 \\
0.00 & 0.00 & 0.00 & 0.00 & 0.00 & 0.03 & 0.22 & 047 & 0.22 & 0.03 \\
0.00 & 0.00 & 0.00 & 0.00 & 0.00 & 0.00 & 0.03 & 022 & 0.47 & 0.21 \\
0.00 & 0.00 & 0.00 & 0.00 & 0.00 & 0.00 & 0.00 & 0.03 & 0.21 & 0.44
\end{array}\right)
$$

Figure 6. The calculated state transition matrix for an initial delta function: the matrix was derived from the Crank-Nicolson scheme with parameters $\Delta t=1, \Delta x=1, D=0.4$ and $K=0.02$

where $\mu_{\mathrm{w}}$ is called the spectral radius of the matrix, and $\lambda_{j}$ represents eigenvalue. The relationship between two measures

$$
\mu_{\mathrm{w}} \leq\|\mathbf{W}\|
$$

is also functional [17], in that the stability of the system can be checked by evaluating the maximum eigenvalue while developing a filter in the matrix form.

\section{Pheromone Filter}

\subsection{Design concept}

Having recognized the result of the stability analysis, the useful design scheme for a smoothing filter is shaped in this section. The filter will be designed based on both the structural characteristics of the model and the analytical results on stability. We call a developed filter pheromone filter.

To design a filter, we first observed a generated matrix $\mathbf{W}$ from Equation (23) in detail, which is generally called the state transition matrix. The calculated matrix is presented in Figure 6. It was sampled after running ten steps for an initial delta spike under the one of the typical conditions. In the figure, the elements marked 0.00 are not precisely zero, but they are pretty small when compared with other numbered elements.

In the matrix, interestingly enough, each row looks similar to the $1 \times 5$ Gaussian kernel. Recall that our revised robot has the similar structure to the one-dimensional filter, which suggests that the pheromone deployment by mobile robots can be modeled straightforwardly from the state transition matrix.

As a first trial, we directly applied the following representative $1 \times 5$ kernel to the pheromone model. 


\begin{tabular}{|l|l|l|l|l|}
\hline$\gamma$ & $\beta$ & $\alpha$ & $\beta$ & $\gamma$
\end{tabular}$\Leftrightarrow$\begin{tabular}{|l|l|l|l|l|}
\hline 0.03 & 0.22 & 0.47 & 0.22 & 0.03 \\
\hline
\end{tabular}

Although our first attempt was inspired from the intuitive observation, the result of the numerical experiment was notable, as shown in Figure 7(a).

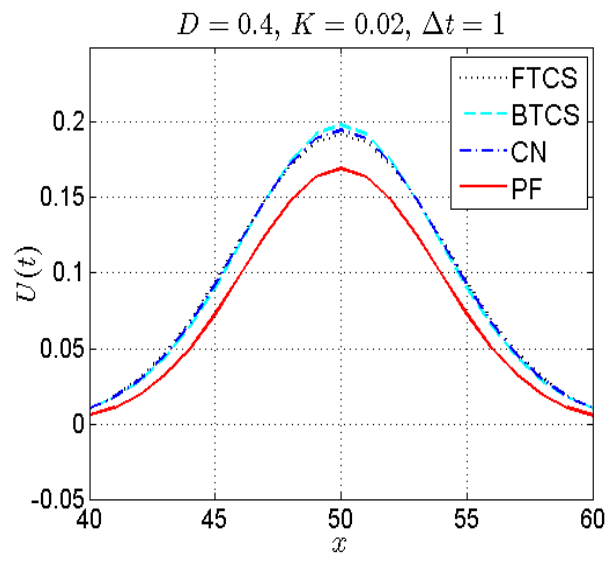

(a) Using $1 \times 5$ kernel

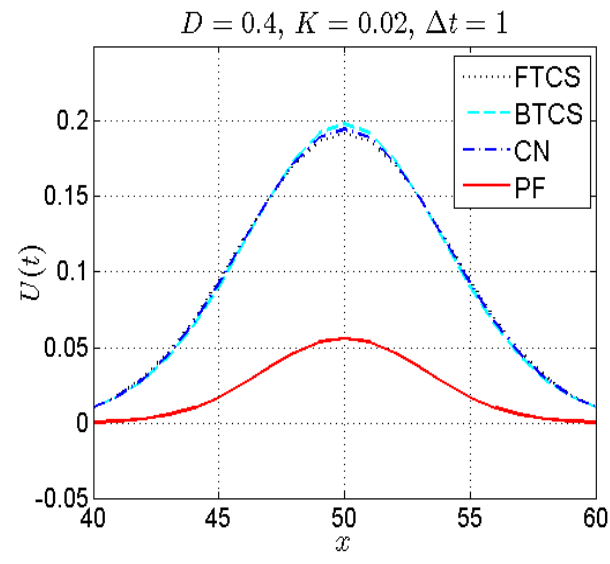

(b) Using $1 \times 3$ kernel

Figure 7. Comparison of the shape of the output potential field using raw kernel: $\mathrm{CN}$ and PF represent the Crank-Nicolson and the Pheromone Filter respectively

The figure was sampled at time step 20 and magnified in order to be precisely observed. Since the $1 \times 3$ kernel is the one more preferred, and is exactly matched with the structure of our revised robot as described in Figure 2, we simply cut off the most end-side entries from Equation (27), i.e., we made

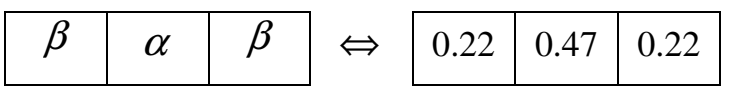

and applied it in the same way as before. The produced potential field is compared in Figure 7(b). As expected from the described operation, the difference in shape to the other methods became larger, whereas the field itself remains stable.

Now, before moving onto the next step, we minimize some inconveniences found in the calculated matrix. That is, from Figure 6, it is indicated that each row of the matrix does not always consist of symmetric kernel. In addition, the diagonal value near the boundary is different to the other diagonal elements. For the latter problem, we can just substitute the major other value for the boundary diagonal one. For example, in Figure 6, the values 0.44 were changed to 0.47 to produce Figure 7 . We then carried out the approximating operation as shown in the following equation. 
International Journal of Computer Science \& Information Technology (IJCSIT) Vol 3, No 5, Oct 2011

$$
\hat{\mathbf{W}}=\left(\begin{array}{ccccc}
\alpha_{1} & \beta_{1} & & \cdots & 0 \\
\beta_{1} & \alpha_{2} & \beta_{2} & & \\
& \ddots & \ddots & \ddots & \\
& & \beta_{m-2} & \alpha_{m-1} & \beta_{m-1} \\
0 & \cdots & & \beta_{m-1} & \alpha_{m}
\end{array}\right) \approx\left(\begin{array}{ccccc}
\alpha_{1} & \beta_{1} & & \cdots & 0 \\
\beta_{2} & \alpha_{2} & \beta_{2} & & \\
& \ddots & \ddots & \ddots & \\
& & \beta_{m-1} & \alpha_{m-1} & \beta_{m-1} \\
0 & \cdots & & \beta_{m} & \alpha_{m}
\end{array}\right)
$$

Next, we adjusted the kernel value of $1 \times 3$ filter to ensure that it kept the total sum equal to or less than that of Equation (27), and also to ensure that it checks the spectral radius of the transition matrix so that the system is kept stable. As a result, a pheromone filter was finally designed as

\begin{tabular}{|c|c|c|c|c|c|c|c|}
\hline$\hat{\beta}$ & $\hat{\alpha}$ & $\hat{\beta}$ & $\Leftrightarrow$ & 0.24 & 0.49 & 0.24 & \\
\hline$(0.49$ & 0.24 & 0.00 & 0.00 & $\ldots$ & & & \\
\hline 0.24 & 0.49 & 0.24 & 0.00 & 0.00 & $\ldots$ & & \\
\hline 0.00 & 0.24 & 0.49 & 0.24 & 0.00 & 0.00 & $\cdots$ & \\
\hline & $\ldots$ & & $\cdots$ & 0.00 & 0.00 & 0.24 & $0.49 \quad 0.24$ \\
\hline & & & & .. & 0.00 & 0.00 & $0.24 \quad 0.49$ \\
\hline
\end{tabular}

We, of course, are aware that the described approximation is not very elegant, and seems to be structured primarily through brute force. Nevertheless, the proposed algorithm is definitely intuitive and not very computationally expensive.

Note that the conventional FTCS scheme can be formed as a filter as well. For example, we can simply rearrange Equation (3) as

$$
U_{i}^{n+1}=a U_{i-1}^{n}+b U_{i}^{n}+a U_{i+1}^{n},
$$

where $a=\frac{D \Delta t}{(\Delta x)^{2}}, \quad b=1-\Delta t K-\frac{2 D \Delta t}{(\Delta x)^{2}}$, and obviously, when seen in this way, it is easy to implement on a real robot. We now have more or less the same structure, yet we also have a stability-guaranteed filter.

For the approximated $100 \times 100$ transition matrix calculated using the finally designed filter, $\mu_{\mathrm{w}}$ was calculated as 0.97 , which means that the stability of the systems is secured.

\subsection{Results and discussion}

Using the finally designed filter, the initial pheromone of delta spike was deployed in order to observe the propagation of the potential field. Figure 8 shows the enlarged shape of the produced potential field and its temporal propagation. In Figure 8(a), the shape agrees reasonably well with the other well-known numerical solvers. Note that, compared with Figure 7, the shape is adjusted to be closer to that generated by the other numerical solvers, and the tuning process is relatively simple as mentioned in the previous section. Although the ultimate purpose may be somewhat different, the same idea is applicable to the resizing of the Gaussian smoothing filter for an image plane. In addition, the pheromone is propagated in a stable and gradual way, which is shown in Figure 8(b). 
From these results, the designed filter seems to provide an efficient way of producing a stable potential field. It should be noted that each scalar value of the pheromone filter is virtually identical to each element of our model shown in Figure 2, which implies that the revised mobile robot could play the role of the diffusion filter of the pheromone through the RFID tags distributed in an environment.

To evaluate the proposed algorithm more quantitatively, the standard deviation of each potential field is compared in Table 1. Since the pheromone potential field eventually disappears due to evaporation, only a forequarter out of the whole process is being compared. From the table, it is again observed that the proposed pheromone filter (PFM) works in nearly the same way as the FTCS scheme, which is the closest numerical method in accuracy to the differential pheromone model equation.

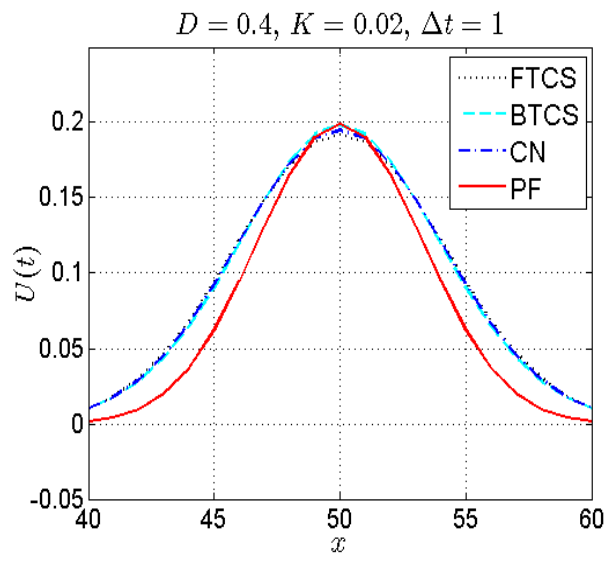

(a) Sampled at step 20

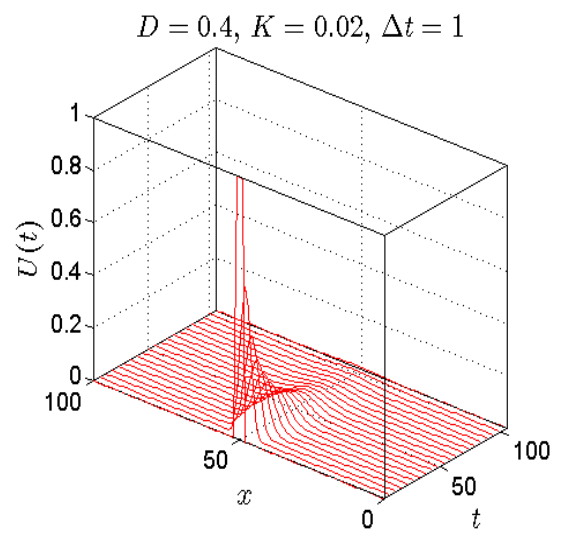

(b) Temporal propagation

Figure 8 . Pheromone potential field generated using the $1 \times 3$ modified pheromone filter

Table 1. Standard deviation of each potential field for given steps: PFR and PFM represent Pheromone Filter Raw (Equation (28)) and Pheromone Filter Modified (Equation (30)), respectively

\begin{tabular}{|l|c|c|c|c|c|}
\hline \multirow{2}{*}{ Method } & \multicolumn{5}{|c|}{ Stdev. for steps $(\times 100)$} \\
\cline { 2 - 6 } & 10 & 20 & 30 & 40 & 50 \\
\hline FTCS & 7.14 & 4.85 & 3.53 & 2.64 & 2.02 \\
\hline PFR & 3.89 & 1.27 & 0.44 & 0.16 & 006 \\
\hline PFM & $\mathbf{7 . 3 2}$ & $\mathbf{4 . 5 4}$ & $\mathbf{3 . 0 0}$ & $\mathbf{2 . 0 4}$ & $\mathbf{1 . 4 1}$ \\
\hline
\end{tabular}

\section{Conclusions}

This paper has formulated the stability condition for the pheromone potential field. The established criterion can provide a general guideline for researchers in the relevant field. Based on the result of the stability analysis, we further presented a new methodology of making a smoothing kernel, called pheromone filter. The developed method demonstrated stable and accurate performance through the numerical simulation; the stability is guaranteed from the implicit structure and the high-level accuracy was achieved by modification of the kernel elements with observing the output behavior. It is expected that the proposed scheme could provide a practical technique for designing a filtering system wherein the stability of the system is secured. 
International Journal of Computer Science \& Information Technology (IJCSIT) Vol 3, No 5, Oct 2011

Despite these advantages, the proposed filter has yet to be evaluated in a batch manner. We are developing a sequential filtering algorithm, which is required in order to identify and update the kernel value online.

Last but not least, it is worth noting that an automatic tuning method for each element of the filter can make the method more elegant and practical. We are now planning to implement the presented method on real robots.

\section{REFERENCES}

[1] Wilson, E., Holldobler, B. (1990) The ants, Springer-Verlag.

[2] Theraulaz, G., Bonabeau, E. (1999) "A brief history of stigmergy”, Artificial Life, Vol. 5, No. 1, pp. 97-116.

[3] Payton, D., Daily, M., Estowski, R., Howard, M., Lee, C. (2001) "Pheromone robotics”, Autonomous Robots, Vol. 11, pp. 319-324.

[4] Parunak, H. V. D., Brueckner, S. A., Sauter, J. (2004) "Digital pheromones for coordination of unmanned vehicles", Proc. of Environments for Multi-Agent Systems 2004, pp. 246-264.

[5] Want, R. (2006) “An introduction to RFID technology”, IEEE Pervasive Computing, Vol. 5, No. 1, pp. 25-33.

[6] Mamei, M., Zambonelli, F. (2007) "Pervasive pheromone-based interaction with RFID tags", ACM Transactions on Autonomous and Adaptive Systems, Vol. 2, No. 2, pp. 1-28.

[7] Herianto, Kurabayashi, D. (2009) "Realization of an artificial pheromone system in random data carriers using RFID tags for autonomous navigation", Proc. IEEE International Conference on Robotics and Automation, pp. 2288-2293.

[8] Han, S., Lim, H., Lee, J. (2007) “An efficient localization scheme for a differential-driving mobile robot based on RFID system”, IEEE Transactions on Industrial Electronics, Vol. 54, pp. 3362-3369.

[9] Park, S., Hashimoto, S. (2009) "Autonomous mobile robot navigation using passive RFID in indoor environment”, IEEE Transactions on Industrial Electronics, Vol. 56, pp. 2366-2373.

[10] Vorst, P., Schneegans, S., Yang, B., Zell, A. (2008) "Self-localization with RFID snapshots in densely tagged environments", Proc. IEEE/RSJ International Conference on Intelligent Robots and Systems, pp. 1353-1358.

[11] Kodaka, K., Niwa, H., Sugano, S. (2009) “Active localization of a robot on a lattice of RFID tags by using an entropy map", Proc. IEEE International Conference on Robotics and Automation, pp. 1193-1199.

[12] Camazine, S., Deneubourg, J. -L., Franks, N. R., Sneyd, J., Theraulaz, G., Bonabeau, E. (2001) Self-organization in biological systems, Princeton University Press.

[13] Sugawara, K., Kazama, T., Watanabe, T. (2004) "Foraging behavior of interacting robots with virtual pheromone", Proc. IEEE/RSJ International Conference on Intelligent Robots and Systems, pp. 3074-3079.

[14] Roache, P. J. (1998) Fundamentals of computational fluid dynamics, Hermosa Publishers.

[15] Golub, G. H., Van Loan, C. F. (1996) Matrix computation, The Johns Hopkins University Press.

[16] Crank, J., Nicolson, P. (1996) "A practical method for numerical evaluation of solutions of partial differential equations of the heat conduction type", Advances in Computational Mathematics, Vol. 6, pp. 207-226.

[17] Lancaster, P., Tismenetsky, M. (1985) The theory of matrices, Academic Press. 


\section{Authors}

Piljae Kim completed a BA and an MSc in Marine Engineering from Korea Maritime University. During his PhD course in Mechanical and Control Engineering at the Tokyo Institute of Technology, he achieved another MSc in Artificial Intelligence from The University of Edinburgh. He is currently doing his $\mathrm{PhD}$ at the Tokyo Institute of Technology, in the area of a distributed multi-robot system.

Daisuke Kurabayashi received the BE, ME, and $\mathrm{PhD}$ in the Department of Precision Machinery Engineering, in 1993, 1995, and 1998, respectively from the University of Tokyo. He worked for the Research Institute of Physical and Chemical Research (RIKEN) from 1998 to 2001 as a postdoctoral researcher. Since 2001 , he has been working for the Tokyo Institute of Technology as an associate professor. His research interest includes distributed autonomous systems, functional structure of a network, and bio-robotics. $\mathrm{He}$ is a member of RSJ, JSME, JSPE, SICE and IEEE. 